Research Article

\title{
Strong Convergence Analysis of Iterative Algorithms for Solving Variational Inclusions and Fixed-Point Problems of Pseudocontractive Operators
}

\author{
Zhangsong Yao, ${ }^{1}$ Yan-Kuen Wu $\mathbb{D}^{2},{ }^{2}$ and Ching-Feng Wen $\mathbb{D}^{3,4}$ \\ ${ }^{1}$ School of Information Engineering, Nanjing Xiaozhuang University, Nanjing 211171, China \\ ${ }^{2}$ College of International Business and Shaoxing Key Laboratory of Intelligent Monitoring and Prevention of Smart City, \\ Zhejiang Yuexiu University of Foreign Languages, Shaoxin, Zhejiang, China \\ ${ }^{3}$ Center for Fundamental Science and Research Center for Nonlinear Analysis and Optimization, Kaohsiung Medical University, \\ Kaohsiung 80708, Taiwan \\ ${ }^{4}$ Department of Medical Research, Kaohsiung Medical University Hospital, Kaohsiung 80708, Taiwan
}

Correspondence should be addressed to Yan-Kuen Wu; ykwvnu@gmail.com and Ching-Feng Wen; cfwen@kmu.edu.tw

Received 12 October 2020; Revised 10 November 2020; Accepted 10 March 2021; Published 12 April 2021

Academic Editor: Jen-Chih Yao

Copyright (c) 2021 Zhangsong Yao et al. This is an open access article distributed under the Creative Commons Attribution License, which permits unrestricted use, distribution, and reproduction in any medium, provided the original work is properly cited.

Iterative methods for solving variational inclusions and fixed-point problems have been considered and investigated by many scholars. In this paper, we use the Halpern-type method for finding a common solution of variational inclusions and fixed-point problems of pseudocontractive operators. We show that the proposed algorithm has strong convergence under some mild conditions.

\section{Introduction}

Let $H$ be a real Hilbert space with inner product $\langle\cdot, \cdot\rangle$ and induced norm $\|\cdot\|$. Let $C$ be a nonempty closed and convex subset of $H$. Let $f: C \longrightarrow H$ and $g: H \longrightarrow 2^{H}$ be two nonlinear operators. Recall that the variational inclusion ([1]) is to solve the following problem of finding $x^{\ddagger} \in 2^{H}$ verifying

$$
0 \in(f+g) x^{\ddagger}
$$

Here, use $(f+g)^{-1}(0)$ to denote the set of solutions of (1).

Special Case 1. Let $\delta_{C}: H \longrightarrow\{0,+\infty\}$ be defined by

$$
\delta_{C}= \begin{cases}0, & x \in C, \\ +\infty, & x \notin C .\end{cases}
$$

Setting $g=\partial \delta_{C}$, variational inclusion (1) reduces to find $x^{\ddagger} \in C$ such that

$$
\left\langle f\left(x^{\ddagger}\right), x-x^{\ddagger}\right\rangle \geq 0, \quad \forall x \in C .
$$

Problem (3) is the well-known variational inequality which has been studied, extended, and developed in a broad category of jobs (see, e.g., [2-14]).

Special Case 2. Let $\varphi: H \longrightarrow \mathbb{R} \cup\{+\infty\}$ be a proper lower semicontinuous convex function and $\partial \varphi$ be the subdifferential of $\varphi$. Setting $g=\partial \varphi$, variational inclusion (1) reduces to find $x^{\ddagger} \in H$ such that

$$
\left\langle f\left(x^{\ddagger}\right), x-x^{\ddagger}\right\rangle+\varphi(x)-\varphi\left(x^{\ddagger}\right) \geq 0, \quad \forall x \in H .
$$

Problem (4) is called the mixed quasi-variational inequality [15] which is a very significant extension of variational inequality (3) involving the nonlinear function $\varphi$. It is well known that a large number of practical problems arising in various branches of pure and applied sciences can be formulated as the model of mixed quasi-variational inequality (4) 
Problem (1) plays a key role in minimization, convex feasibility problems, machine learning, and others. A popular algorithm for solving problem (1) is the forwardbackward algorithm [16] generated by

$$
x_{n+1}=(I+\lambda g)^{-1}(I-\lambda f) x_{n}, \quad n \geq 1,
$$

where $I-\lambda f$ is a forward step and $(I+\lambda g)^{-1}$ is a backward step with $\lambda>0$. This algorithm is a splitting algorithm which solves the difficulty of calculating of the resolvent of $f+g$.

Recently, there has been increasing interest for studying common solution problems relevant to (1) (see for example, [17-27]). Especially, Zhao, Sahu, and Wen [28] presented an iterative algorithm for solving a system of variational inclusions involving accretive operators. Ceng and Wen [29] introduced an implicit hybrid steepest-descent algorithm for solving generalized mixed equilibria with variational inclusions and variational inequalities. $\mathrm{Li}$ and Zhao [30] considered an iterate for finding a solution of quasi-variational inclusions and fixed points of nonexpansive mappings.

Motivated by the results in this direction, the main purpose of this paper is to research a common solution problem of variational inclusions and fixed point of pseudocontractions. We suggest a Halpern-type algorithm for solving such problem. We show that the proposed algorithm has strong convergence under some mild conditions.

\section{Preliminaries}

Let $H$ be a real Hilbert space. Let $g: H \longrightarrow 2^{H}$ be an operator. Write $\operatorname{dom}(g)=\{x \in H: g(x) \neq \varnothing\}$. Recall that $g$ is called monotone if $\forall x, y \in \operatorname{dom}(g), u \in g(x)$ and $v \in g(y)$, $\langle x-y, u-v\rangle \geq 0$.

A monotone operator $g$ is maximal monotone if and only if its graph is not strictly contained in the graph of any other monotone operator on $H$.

For a maximal monotone operator $g$ on $H$,

(i) Set $g^{-1} 0=\{x \in H: 0 \in g(x)\}$ (ii) Denote its resolvent by $J_{\lambda}^{g}=(I+\lambda g)^{-1}$ which is single-valued from $H$ into $\operatorname{dom}(g)$

It is known that $g^{-1} 0=\operatorname{Fix}\left(J_{\lambda}^{g}\right), \forall \lambda>0$ and $J_{\lambda}^{g}$ is firmly nonexpansive, i.e.,

$$
\left\|J_{\lambda}^{g} x-J_{\lambda}^{g} y\right\|^{2} \leq\left\langle J_{\lambda}^{g} x-J_{\lambda}^{g} y, x-y\right\rangle,
$$

for all $x, y \in C$.

Let $C$ be a nonempty closed convex subset of a real Hilbert space $H$. Recall that an operator $T: C \longrightarrow C$ is said to be

(i) $L$-Lipschitz if there exists a positive constant $L$ such that

$$
\|T x-T y\| \leq L\|x-y\|, \quad \forall x, y \in C .
$$

If $L=1, T$ is nonexpansive.

(ii) Pseudocontractive if

$$
\langle T x-T y, x-y\rangle \leq\|x-y\|^{2}, \quad \forall x, y \in C .
$$

(iii) Inverse-strongly monotone if

$$
\langle T x-T y, x-y\rangle \geq \alpha\|T x-T y\|^{2}, \quad \forall x, y \in C,
$$

where $\alpha>0$ is a constant and $T$ is also called $\alpha$-ism.

Recall that the projection $P_{C}$ is an orthographic projection from $H$ onto $C$, which is defined by $\left\|x-P_{C}(x)\right\|=\min _{y \in C}\|x-y\|$. It is known that $P_{C}$ is nonexpansive.

Lemma 1 (see $[23,31])$. Let $C$ be a nonempty closed convex subset of a real Hilbert space $H$. Let $T: C \longrightarrow C$ be an L-Lipschitz pseudocontractive operator. Then,

(i) $T$ is demiclosed, i.e., $x_{n} \rightarrow p$ and $T\left(x_{n}\right) \longrightarrow q \Rightarrow T$ $(p)=q$

(ii) For $0<\zeta<\left(1 /\left(\sqrt{1+L^{2}}+1\right)\right), \forall x \in C$ and $y \in$ Fix $(T)$, we have

$$
\|T[(1-\zeta) x+\zeta T(x)]-y\|^{2} \leq\|x-y\|^{2}+(1-\zeta)\|x-T[(1-\zeta) x+\zeta T(x)]\|^{2} .
$$

Lemma 2 (see [16, 32]). Let $H$ be a real Hilbert space and let $g$ be a maximal monotone operator on $H$. Then, we have

$$
\left\|J_{s}^{g}(x)-J_{t}^{g}(x)\right\|^{2} \leq \frac{s-t}{t}\left\langle J_{s}^{g}(x)-J_{t}^{g}(x), J_{s}^{g}(x)-x\right\rangle,
$$

for all $s, t>0$ and $x \in H$.

Lemma 3 (see [33]). Assume that a real number sequence $\left\{a_{n}\right\} \subset[0, \infty)$ satisfies

$$
a_{n+1} \leq\left(1-\gamma_{n}\right) a_{n}+\delta_{n} \gamma_{n}
$$

where $\left\{\gamma_{n}\right\} \subset(0,1)$ and $\left\{\delta_{n}\right\} \subset(-\infty,+\infty)$ satisfy the following conditions:

(i) $\sum_{n=1}^{\infty} \gamma_{n}=\infty$

(ii) $\lim \sup _{n \rightarrow \infty} \delta_{n} \leq 0$ or $\sum_{n=1}^{\infty}\left|\delta_{n} \gamma_{n}\right|<\infty$

Then, $\lim _{n \longrightarrow \infty} a_{n}=0$.

Lemma 4 (see [8]). Let $\left\{s_{n}\right\} \subset(0, \infty)$ be a sequence. Assume that there exists at least a subsequence $\left\{s_{n_{i}}\right\}$ of $\left\{s_{n}\right\}$ verifying $s_{n_{i}} \leq s_{n_{i}+1}$ for all $i \geq 0$. Let $\{\tau(n)\}$ be an integer sequence defined as $\tau(n)=\max \left\{i \leq n: s_{n_{i}}<s_{n_{i}+1}\right\}$. Then $\tau(n) \longrightarrow \infty$ as $n \longrightarrow \infty$ and 


$$
\max \left\{s_{\tau(n)}, s_{n}\right\} \leq s_{\tau(n)+1} .
$$

\section{Main Results}

Let $C$ be a nonempty closed convex subset of a real Hilbert space $H$. Let the operator $f: C \longrightarrow H$ be an $\alpha$-ism. Let $g: H \longrightarrow 2^{H}$ be a maximal monotone operator with $\operatorname{dom}(g) \subset C$. Let $T: C \longrightarrow C$ be an $L$-Lipschitz pseudocontractive operator with $L>1$. Let $\left\{\alpha_{n}\right\} \subset(0,1)$ and $\left\{\lambda_{n}\right\} \subset(0, \infty)$ be two sequences. Let $v$ and $\zeta$ be two constants.

Next, we introduce a Halpern-type algorithm for finding a common solution of variational inclusion (1) and fixed point of pseudocontractive operator $T$.

Algorithm 1. Let $u \in C$ be a fixed point. Choose $x_{0} \in C$. Set $n=0$.

Step 1. For given $x_{n}$, compute $y_{n}$ by

$$
y_{n}=(1-\nu) x_{n}+\nu T\left((1-\zeta) x_{n}+\zeta T x_{n}\right)
$$

Step 2. Compute $x_{n+1}$ by

$$
x_{n+1}=\alpha_{n} u+\left(1-\alpha_{n}\right) J_{\lambda_{n}}^{g}\left(I-\lambda_{n} f\right) y_{n} .
$$

Step 3. Set $n:=n+1$ and return to Step 1 .

Next, we prove the convergence of Algorithm 1.

Theorem 1. Suppose that $\Gamma$ : $=$ Fix $(T) \cap(f+g)^{-1}(0) \neq \varnothing$. Assume that the following conditions are satisfied:

$$
\begin{aligned}
& \lim _{n \longrightarrow \infty} \alpha_{n}=0 \text { and } \sum_{n=1}^{\infty} \alpha_{n}=\infty \\
& 0<d_{1}<\lambda_{n}<d_{2}<2 \alpha \text { and } 0<v<\zeta<\left(1 /\left(\sqrt{1+L^{2}}+1\right)\right)
\end{aligned}
$$

Then, the sequence $\left\{x_{n}\right\}$ generated by Algorithm 1 converges strongly to $P_{\Gamma}(u)$.

Proof. Let $x^{*} \in \operatorname{Fix}(T) \cap(f+g)^{-1}(0)$. Set $u_{n}=J_{\lambda_{n}}^{g}\left(I-\lambda_{n}\right.$ f) $y_{n}, \quad \forall n \geq 0$. Since $f$ is $\alpha$-ism, we have

$$
\left\langle f\left(y_{n}\right)-f\left(x^{*}\right), y_{n}-x^{*}\right\rangle \geq \alpha\left\|f\left(y_{n}\right)-f\left(x^{*}\right)\right\|^{2} .
$$

By the nonexpansivity of $J_{\lambda_{n}}^{g}$, we have

$$
\begin{aligned}
\left\|u_{n}-x^{*}\right\|^{2} & =\left\|J_{\lambda_{n}}^{g}\left(I-\lambda_{n} f\right) y_{n}-J_{\lambda_{n}}^{g}\left(I-\lambda_{n} f\right) x^{*}\right\|^{2} \\
& \leq\left\|y_{n}-x^{*}-\lambda_{n}\left[f\left(y_{n}\right)-f\left(x^{*}\right)\right]\right\|^{2} \\
& =\left\|y_{n}-x^{*}\right\|^{2}-2 \lambda_{n}\left\langle f\left(y_{n}\right)-f\left(x^{*}\right), y_{n}-x^{*}\right\rangle+\lambda_{n}^{2}\left\|f\left(y_{n}\right)-f\left(x^{*}\right)\right\|^{2} \\
& \leq\left\|y_{n}-x^{*}\right\|^{2}-2 \lambda_{n} \alpha\left\|f\left(y_{n}\right)-f\left(x^{*}\right)\right\|^{2}+\lambda_{n}^{2}\left\|f\left(y_{n}\right)-f\left(x^{*}\right)\right\|^{2} \\
& =\left\|y_{n}-x^{*}\right\|^{2}-\lambda_{n}\left(2 \alpha-\lambda_{n}\right)\left\|f\left(y_{n}\right)-f\left(x^{*}\right)\right\|^{2} \\
& \leq\left\|y_{n}-x^{*}\right\|^{2}-d_{1}\left(2 \alpha-d_{2}\right)\left\|f\left(y_{n}\right)-f\left(x^{*}\right)\right\|^{2}(\text { by condition }(r 2)) \\
& \leq\left\|y_{n}-x^{*}\right\|^{2} .
\end{aligned}
$$

Using Lemma 1, we get

$$
\left\|T((1-\zeta) I+\zeta T) x_{n}-x^{*}\right\|^{2} \leq\left\|x_{n}-x^{*}\right\|^{2}+(1-\zeta)\left\|x_{n}-T((1-\zeta) I+\zeta T) x_{n}\right\|^{2}
$$

This together with (14) implies that

$$
\begin{aligned}
\left\|y_{n}-x^{*}\right\|^{2}= & \left\|(1-v) x_{n}+\nu T((1-\zeta) I+\zeta T) x_{n}-x^{*}\right\|^{2} \\
= & \left\|(1-v)\left(x_{n}-x^{*}\right)+\nu\left(T((1-\zeta) I+\zeta T) x_{n}-x^{*}\right)\right\|^{2} \\
= & (1-v)\left\|x_{n}-x^{*}\right\|^{2}+\nu\left\|T((1-\zeta) I+\zeta T) x_{n}-x^{*}\right\|^{2} \\
& -v(1-v)\left\|T((1-\zeta) I+\zeta T) x_{n}-x_{n}\right\|^{2} \\
\leq & \left\|x_{n}-x^{*}\right\|^{2}-v(\zeta-v)\left\|T((1-\zeta) I+\zeta T) x_{n}-x_{n}\right\|^{2} \\
\leq & \left\|x_{n}-x^{*}\right\|^{2} .
\end{aligned}
$$

According to (15)-(19), we obtain

$$
\begin{aligned}
\left\|x_{n+1}-x^{*}\right\| & =\left\|\alpha_{n}\left(u-x^{*}\right)+\left(1-\alpha_{n}\right)\left(u_{n}-x^{*}\right)\right\| \\
& \leq \alpha_{n}\left\|u-x^{*}\right\|+\left(1-\alpha_{n}\right)\left\|x_{n}-x^{*}\right\| \\
& \leq \cdots \\
& \leq \max \left\{\left\|u-x^{*}\right\|,\left\|x_{0}-x^{*}\right\|\right\} .
\end{aligned}
$$

Then, the sequence $\left\{x_{n}\right\}$ is bounded. The sequences $\left\{u_{n}\right\}$ and $\left\{y_{n}\right\}$ are also bounded.

Again, by (15)-(19), we deduce 


$$
\begin{aligned}
\left\|x_{n+1}-x^{*}\right\|^{2} & =\left\|\alpha_{n}\left(u-x^{*}\right)+\left(1-\alpha_{n}\right)\left(u_{n}-x^{*}\right)\right\|^{2} \\
& \left.\leq \alpha_{n}\left\|u-x^{*}\right\|^{2}+\left(1-\alpha_{n}\right)\left\|u_{n}-x^{*}\right\|^{2} \text { (by the convexity of }\|\cdot\|^{2}\right) \\
& \leq \alpha_{n}\left\|u-x^{*}\right\|^{2}+\left\|u_{n}-x^{*}\right\|^{2} \\
& \leq \alpha_{n}\left\|u-x^{*}\right\|^{2}+\left\|x_{n}-x^{*}\right\|^{2}-d_{1}\left(2 \alpha-d_{2}\right)\left\|f\left(y_{n}\right)-f\left(x^{*}\right)\right\|^{2}-\nu(\zeta-v)\left\|T((1-\zeta) I+\zeta T) x_{n}-x_{n}\right\|^{2} .
\end{aligned}
$$

It follows that

$$
d_{1}\left(2 \alpha-d_{2}\right)\left\|f\left(y_{n}\right)-f\left(x^{*}\right)\right\|^{2}+\nu(\zeta-v)\left\|T((1-\zeta) I+\zeta T) x_{n}-x_{n}\right\|^{2} \leq \alpha_{n}\left\|u-x^{*}\right\|^{2}+\left\|x_{n}-x^{*}\right\|^{2}-\left\|x_{n+1}-x^{*}\right\|^{2} .
$$

Since $J_{\lambda_{n}}^{g}$ is firmly nonexpansive, using (6), we have

$$
\begin{aligned}
\left\|u_{n}-x^{*}\right\|^{2} & =\left\|J_{\lambda_{n}}^{g}\left(I-\lambda_{n} f\right) y_{n}-J_{\lambda_{n}}^{g}\left(I-\lambda_{n} f\right) x^{*}\right\|^{2} \\
& \leq\left\langle\left(I-\lambda_{n} f\right) y_{n}-\left(I-\lambda_{n} f\right) x^{*}, u_{n}-x^{*}\right\rangle \\
& =\left\langle y_{n}-x^{*}, u_{n}-x^{*}\right\rangle-\lambda_{n}\left\langle u_{n}-x^{*}, f\left(y_{n}\right)-f\left(x^{*}\right)\right\rangle \\
& =\frac{1}{2}\left(\left\|y_{n}-x^{*}\right\|^{2}+\left\|u_{n}-x^{*}\right\|^{2}-\left\|y_{n}-u_{n}\right\|^{2}\right)-\lambda_{n}\left\langle y_{n}-x^{*}, f\left(y_{n}\right)-f\left(x^{*}\right)\right\rangle-\lambda_{n}\left\langle u_{n}-y_{n}, f\left(y_{n}\right)-f\left(x^{*}\right)\right\rangle \\
& \leq \frac{1}{2}\left(\left\|y_{n}-x^{*}\right\|^{2}+\left\|u_{n}-x^{*}\right\|^{2}-\left\|y_{n}-u_{n}\right\|^{2}\right)+\lambda_{n}\left\|u_{n}-y_{n}\right\|\left\|f\left(y_{n}\right)-f\left(x^{*}\right)\right\|,
\end{aligned}
$$

which leads to

$$
\begin{aligned}
\left\|u_{n}-x^{*}\right\|^{2} & \leq\left\|y_{n}-x^{*}\right\|^{2}-\left\|y_{n}-u_{n}\right\|^{2}+2 \lambda_{n}\left\|u_{n}-y_{n}\right\|\left\|f\left(y_{n}\right)-f\left(x^{*}\right)\right\| \\
& \leq\left\|x_{n}-x^{*}\right\|^{2}-\left\|y_{n}-u_{n}\right\|^{2}+2 \lambda_{n}\left\|u_{n}-y_{n}\right\|\left\|f\left(y_{n}\right)-f\left(x^{*}\right)\right\| .
\end{aligned}
$$

Combining (21) with (24), we obtain

$$
\left\|x_{n+1}-x^{*}\right\|^{2} \leq \alpha_{n}\left\|u-x^{*}\right\|^{2}+\left\|u_{n}-x^{*}\right\|^{2} \leq \alpha_{n}\left\|u-x^{*}\right\|^{2}+\left\|x_{n}-x^{*}\right\|^{2}-\left\|y_{n}-u_{n}\right\|^{2}+2 \lambda_{n}\left\|u_{n}-y_{n}\right\|\left\|f\left(y_{n}\right)-f\left(x^{*}\right)\right\| .
$$

which results in that

$$
\left\|y_{n}-u_{n}\right\|^{2} \leq \alpha_{n}\left\|u-x^{*}\right\|^{2}+\left\|x_{n}-x^{*}\right\|^{2}-\left\|x_{n+1}-x^{*}\right\|^{2}+2 \lambda_{n}\left\|u_{n}-y_{n}\right\|\left\|f\left(y_{n}\right)-f\left(x^{*}\right)\right\| .
$$

Next, we analyze two cases. $\exists n_{0} \in \mathbb{N}$ such that $\left\|x_{n+1}-x^{*}\right\| \leq\left\|x_{n}-x^{*}\right\|, \forall n \geq n_{0}$. (ii) For any $n_{0} \in \mathbb{N}, \exists m \geq n_{0}$ such that $\left\|x_{m}-x^{*}\right\| \leq\left\|x_{m+1}-x^{*}\right\|$.

In case of (i), $\lim _{n \longrightarrow \infty}\left\|x_{n}-x^{*}\right\|$ exists. From (22), we deduce

$$
\lim _{n \longrightarrow \infty}\left\|f\left(y_{n}\right)-f\left(x^{*}\right)\right\|=0
$$

and

$$
\lim _{n \longrightarrow \infty}\left\|T((1-\zeta) I+\zeta T) x_{n}-x_{n}\right\|=0 .
$$

It follows from (14) that 
$\lim _{n \longrightarrow \infty}\left\|y_{n}-x_{n}\right\|=\lim _{n \longrightarrow \infty} v\left\|T((1-\zeta) I+\zeta T) x_{n}-x_{n}\right\|=0$.

On the basic of (26) and (27), we have $\lim _{n \longrightarrow \infty}\left\|y_{n}-u_{n}\right\|=\lim _{n \longrightarrow \infty}\left\|y_{n}-J_{\lambda_{n}}^{g}\left(I-\lambda_{n} f\right) y_{n}\right\|=0$.
Note that $\left\|x_{n+1}-x_{n}\right\| \leq \alpha_{n}\left\|u-x_{n}\right\|+\left(1-\alpha_{n}\right)\left\|u_{n}-x_{n}\right\|$. Thanks to (29) and (30), we derive that

$$
\lim _{n \longrightarrow \infty}\left\|x_{n+1}-x_{n}\right\|=0 .
$$

However,

$$
\begin{aligned}
\left\|x_{n}-T x_{n}\right\| & \leq\left\|T((1-\zeta) I+\zeta T) x_{n}-x_{n}\right\|+\left\|T x_{n}-T((1-\zeta) I+\zeta T) x_{n}\right\| \\
& \leq\left\|T((1-\zeta) I+\zeta T) x_{n}-x_{n}\right\|+\zeta L\left\|x_{n}-T x_{n}\right\|,
\end{aligned}
$$

We have

$$
\left\|x_{n}-T x_{n}\right\| \leq \frac{1}{1-\zeta L}\left\|T((1-\zeta) I+\zeta T) x_{n}-x_{n}\right\| .
$$

This together with (28) implies that

$$
\lim _{n \longrightarrow \infty}\left\|x_{n}-T x_{n}\right\|=0 .
$$

Set $p=P_{\Gamma}(u)$. Next, we prove that

$$
\lim \sup _{n \longrightarrow \infty}\left\langle u-p, x_{n+1}-p\right\rangle \leq 0 .
$$

Since $\left\{x_{n+1}\right\}$ is bounded, there exists a subsequence $\left\{x_{n_{i}+1}\right\}$ of $\left\{x_{n+1}\right\}$ satisfying
(1) $x_{n_{i}+1}-\tilde{x}$ (hence, $x_{n_{i}}-\tilde{x}$ by (31))

(2) $\lim \sup _{n \rightarrow \infty}\left\langle u-p, x_{n+1}-p\right\rangle=\lim _{i \rightarrow \infty}\langle u-$ $\left.p, x_{n_{i}+1}-p\right\rangle$

From (34) and Lemma 1, we obtain $\tilde{x} \in \operatorname{Fix}(T)$. Owing to (29) and (30), we have that $y_{n_{i}}-\tilde{x}$ and

$$
\lim _{i \rightarrow \infty}\left\|J_{\lambda_{n_{i}}}^{g}\left(I-\lambda_{n_{i}} f\right) y_{n_{i}}-y_{n_{i}}\right\|=0 .
$$

Since $\lambda_{n} \in\left(d_{1}, d_{2}\right)$, without loss of generality, we assume that $\lambda_{n_{i}} \longrightarrow \lambda^{\dagger}>0(i \longrightarrow \infty)$. Observe that

$$
\begin{aligned}
& \left\|J_{\lambda_{n_{i}}^{g}}^{g}\left(I-\lambda_{n_{i}} f\right) y_{n_{i}}-J_{\lambda^{\dagger}}^{g}\left(I-\lambda^{\dagger} f\right) y_{n_{i}}\right\| \leq\left\|J_{\lambda_{n_{i}}}^{g}\left(I-\lambda_{n_{i}} f\right) y_{n_{i}}-J_{\lambda^{\dagger}}^{g}\left(I-\lambda_{n_{i}} f\right) y_{n_{i}}\right\| \\
& \quad+\left\|J_{\lambda^{\dagger}}^{g}\left(I-\lambda_{n_{i}} f\right) y_{n_{i}}-J_{\lambda^{\dagger}}^{g}\left(I-\lambda^{\dagger} f\right) y_{n_{i}}\right\| \\
& \leq\left\|J_{\lambda_{n_{i}}}^{g}\left(I-\lambda_{n_{i}} f\right) y_{n_{i}}-J_{\lambda^{\dagger}}^{g}\left(I-\lambda_{n_{i}} f\right) y_{n_{i}}\right\|+\mid \lambda_{n_{i}}-\lambda^{\dagger}\left\|f\left(y_{n_{i}}\right)\right\| .
\end{aligned}
$$

Applying Lemma 2, we obtain

$$
\begin{aligned}
& \left\|J_{\lambda_{n_{i}}}^{g}\left(I-\lambda_{n_{i}} f\right) y_{n_{i}}-J_{\lambda^{\dagger}}^{g}\left(I-\lambda_{n_{i}} f\right) y_{n_{i}}\right\|^{2} \\
& \leq \frac{\lambda_{n_{i}}-\lambda^{\dagger}}{\lambda^{\dagger}}\left\langle J_{\lambda_{n_{i}}}^{g}\left(I-\lambda_{n_{i}} f\right) y_{n_{i}}-J_{\lambda^{\dagger}}^{g}\left(I-\lambda_{n_{i}} f\right) y_{n_{i}}, J_{\lambda_{n_{i}}}^{g}\left(I-\lambda_{n_{i}} f\right) y_{n_{i}}-\left(I-\lambda_{n_{i}} f\right) y_{n_{i}}\right\rangle \\
& \leq \frac{\left|\lambda_{n_{i}}-\lambda^{\dagger}\right|}{\lambda^{\dagger}}\left\|J_{\lambda_{n_{i}}}^{g}\left(I-\lambda_{n_{i}} f\right) y_{n_{i}}-J_{\lambda^{\dagger}}^{g}\left(I-\lambda_{n_{i}} f\right) y_{n_{i}}\right\|\left\|J_{\lambda_{n_{i}}}^{g}\left(I-\lambda_{n_{i}} f\right) y_{n_{i}}-\left(I-\lambda_{n_{i}} f\right) y_{n_{i}}\right\| .
\end{aligned}
$$

It follows that

$$
\left\|J_{\lambda_{n_{i}}}^{g}\left(I-\lambda_{n_{i}} f\right) y_{n_{i}}-J_{\lambda^{\dagger}}^{g}\left(I-\lambda_{n_{i}} f\right) y_{n_{i}}\right\| \leq \frac{\left|\lambda_{n_{i}}-\lambda^{\dagger}\right|}{\lambda^{\dagger}}\left\|J_{\lambda_{n_{i}}}^{g}\left(I-\lambda_{n_{i}} f\right) y_{n_{i}}-\left(I-\lambda_{n_{i}} f\right) y_{n_{i}}\right\| .
$$


Thanks to (37) and (39), we get

$$
\left\|J_{\lambda_{n_{i}}}^{g}\left(I-\lambda_{n_{i}} f\right) y_{n_{i}}-J_{\lambda^{\dagger}}^{g}\left(I-\lambda^{\dagger} f\right) y_{n_{i}}\right\| \leq\left|\lambda_{n_{i}}-\lambda^{\dagger}\right|\left\|f\left(y_{n_{i}}\right)\right\|+\frac{\left|\lambda_{n_{i}}-\lambda^{\dagger}\right|}{\lambda^{\dagger}}\left\|J_{\lambda_{n_{i}}}^{g}\left(I-\lambda_{n_{i}} f\right) y_{n_{i}}-\left(I-\lambda_{n_{i}} f\right) y_{n_{i}}\right\| .
$$
get

Noting that $\lambda_{n_{i}} \longrightarrow \lambda^{\dagger}(i \longrightarrow \infty)$, from (36) and (40), we

$$
\lim _{i \rightarrow \infty}\left\|y_{n_{i}}-J_{\lambda^{\dagger}}^{g}\left(I-\lambda^{\dagger} f\right) y_{n_{i}}\right\|=0
$$

By Lemma 1, we deduce that $\tilde{x} \in \operatorname{Fix}\left(J_{\lambda^{\dagger}}^{g}\left(I-\lambda^{\dagger} f\right)\right)=$ $(f+g)^{-1}(0)$. Therefore, $\tilde{x} \in \Gamma$ and

$$
\lim \sup _{n \longrightarrow \infty}\left\langle u-p, x_{n+1}-p\right\rangle=\lim _{i \longrightarrow \infty}\left\langle u-p, x_{n_{i}+1}-p\right\rangle=\langle u-p, \tilde{x}-p\rangle \leq 0
$$

From (15), we have

$$
\begin{aligned}
\left\|x_{n+1}-p\right\|^{2} & =\left\|\alpha_{n}(u-p)+\left(1-\alpha_{n}\right)\left(u_{n}-p\right)\right\|^{2} \\
& \leq\left(1-\alpha_{n}\right)\left\|u_{n}-p\right\|^{2}+2 \alpha_{n}\left\langle u-p, x_{n+1}-p\right\rangle \\
& \leq\left(1-\alpha_{n}\right)\left\|x_{n}-p\right\|^{2}+2 \alpha_{n}\left\langle u-p, x_{n+1}-p\right\rangle .
\end{aligned}
$$

Applying Lemma 3 to (43) to deduce $x_{n} \longrightarrow p$.

In case of (ii), let $s_{n}=\left\{\left\|x_{n}-x^{*}\right\|\right\}$. So, we have $s_{n_{0}} \leq s_{n_{0}+1}$. Define an integer sequence $\{\tau(n)\}, \forall n \geq n_{0}$, by $\tau(n)=\max$ $\left\{i \in \mathbb{N} \mid n_{0} \leq i \leq n, s_{i} \leq s_{i+1}\right\}$. It is obvious that $\lim _{n \rightarrow \infty} \tau(n)=$ $\infty$ and $s_{\tau(n)} \leq s_{\tau(n)+1}$ for all $n \geq n_{0}$. Similarly, we can prove that $\lim _{n \longrightarrow \infty}\left\|x_{\tau(n)}-T x_{\tau(n)}\right\|=0$ and $\lim _{n \longrightarrow \infty} \| J_{\lambda_{\tau(n)}}^{g}$ (I$\left.\lambda_{\tau(n)} f\right) x_{\tau(n)} \|=0$. Therefore, all weak cluster points $\omega_{w}\left(x_{\tau(n)}\right) \subset \Gamma$. Consequently,

$$
\lim \sup _{n \longrightarrow \infty}\left\langle u-p, x_{\tau(n)}-p\right\rangle \leq 0 .
$$

Note that $s_{\tau(n)} \leq s_{\tau(n)+1}$. From (43), we deduce

$$
s_{\tau(n)}^{2} \leq s_{\tau(n)+1}^{2} \leq\left(1-\alpha_{\tau(n)}\right) s_{\tau(n)}^{2}+2 \alpha_{\tau(n)}\left\langle u-p, x_{\tau(n)+1}-p\right\rangle .
$$

It follows that

$$
s_{\tau(n)}^{2} \leq 2\left\langle u-p, x_{\tau(n)+1}-p\right\rangle .
$$

Combining (44) and (46), we have $\lim \sup _{n \longrightarrow \infty} s_{\tau(n)} \leq 0$ and hence

$$
\lim _{k \longrightarrow \infty} s_{\tau(k)}=0 .
$$

From (45), we deduce that $\lim \sup _{n \rightarrow \infty} s_{\tau(n)+1}^{2} \leq$ $\lim \sup _{n \longrightarrow \infty} s_{\tau(n)}^{2}$. This together with (47) implies that $\lim _{n \longrightarrow \infty} s_{\tau(n)+1}=0$. According to Lemma 4, we get $0 \leq s_{n} \leq \max \left\{s_{\tau(n)}, s_{\tau(n)+1}\right\}$. Therefore, $s_{n} \longrightarrow 0$ and $x_{n} \longrightarrow p$. This completes the proof.

Remark 1. Since the pseudocontractive operator is nonexpansive, Theorem 1 still holds if $T$ is nonexpansive.
Remark 2. Assumption ( $r 1)$ imposed on parameter $\alpha_{n}$ is essential and we do not add any other assumptions.

\section{Data Availability}

No data were used to support this study.

\section{Conflicts of Interest}

The authors declare that they have no conflicts of interest.

\section{Acknowledgments}

Zhangsong Yao was partially supported by the Grant 19KJD100003. Ching-Feng Wen was partially supported by the Grant of MOST 109-2115-M-037-001.

\section{References}

[1] Y.-P. Fang and N.-J. Huang, "H-Monotone operator and resolvent operator technique for variational inclusions," Applied Mathematics and Computation, vol. 145, no. 2-3, pp. 795-803, 2003.

[2] L. C. Ceng, "Composite implicit viscosity extragradient algorithms for systems of variational inequalities with fixed point constraints of asymptotically nonexpansive mappings," Applied Analysis and Optimization, vol. 3, pp. 423-437, 2019.

[3] L.-C. Ceng, A. Petrusel, J.-C. Yao, and Y. Yao, "Hybrid viscosity extragradient method for systems of variational inequalities, fixed points of nonexpansive mappings, zero points of accretive operators in Banach spaces," Fixed Point Theory, vol. 19, no. 2, pp. 487-502, 2018.

[4] L. C. Ceng, A. Petrusel, J. C. Yao, and Y. Yao, "Systems of variational inequalities with hierarchical variational inequality constraints for Lipschitzian pseudocontractions," Fixed Point Theory, vol. 20, pp. 113-133, 2019.

[5] Q. L. Dong, Y. Peng, and Y. Yao, "Alternated inertial projection methods for the split equality problem," Journal of Nonlinear and Convex Analysis, vol. 22, pp. 53-67, 2021.

[6] P. Hartman and G. Stampacchia, "On some non-linear elliptic differential-functional equations," Acta Mathematica, vol. 115, pp. 271-310, 1966.

[7] X.-H. Li, Q.-L. Dong, and A. Gibali, "Some new iterative algorithms for finding fixed points of nonexpansive 
mappings," Journal of Optimization Theory and Applications, vol. 2, pp. 143-154, 2020.

[8] P. E. Maingé and M. L. Gobinddass, "Convergence of one-step projected gradient methods for variational inequalities," Journal of Optimization Theory and Applications, vol. 171, no. 1, pp. 146-168, 2016.

[9] N. Shahzad and H. Zegeye, "Convergence theorems of common solutions for fixed point, variational inequality and equilibrium problems," Journal of Nonlinear and Variational Analysis, vol. 3, pp. 189-203, 2019.

[10] F. Wang and H. Pham, "On a new algorithm for solving variational inequality and fixed point problems," Journal of Nonlinear and Convex Analysis, vol. 3, pp. 225-233, 2019.

[11] Y. Yao, Y.-C. Liou, and J.-C. Yao, "Iterative algorithms for the split variational inequality and fixed point problems under nonlinear transformations," The Journal of Nonlinear Sciences and Applications, vol. 10, no. 2, pp. 843-854, 2017.

[12] Y. Yao, M. Postolache, and J. C. Yao, "Iterative algorithms for generalized varational inequalities," University Politehnica of Bucharest Scientific Bulletin-Series A-Applied Mathematics and Physics, vol. 81, pp. 3-16, 2019.

[13] X. Zhao and Y. Yao, "Modified extragradient algorithms for solving monotone variational inequalities and fixed point problems," Optimization, vol. 69, no. 9, pp. 1987-2002, 2020.

[14] L. J. Zhu, Y. Yao, and M. Postolache, "Projection methods with linesearch technique for pseudomonotone equilibrium problems and fixed point problems," Politehnica University of Bucharest. Series A, vol. 83, no. 1, pp. 3-14, 2021.

[15] M. A. Noor, "Generalized set-valued variational inclusions and resolvent equations," Journal of Mathematical Analysis and Applications, vol. 228, no. 1, pp. 206-220, 1998.

[16] H. H. Bauschke and P. L. Combettes, Convex Analysis and Monotone Operator Theory in Hilbert Spaces, Springer, Berlin, Germany, 2011.

[17] P. Cholamjiak, S. Suantai, and P. Sunthrayuth, "An explicit parallel algorithm for solving variational inclusion problem and fixed point problem in Banach spaces," Banach Journal of Mathematical Analysis, vol. 14, no. 1, pp. 20-40, 2020.

[18] W. Cholamjiak, S. A. Khan, D. Yambangwai, and K. R. Kazmi, "Strong convergence analysis of common variational inclusion problems involving an inertial parallel monotone hybrid method for a novel application to image restoration," Revista de la Real Academia de Ciencias Exactas, Físicas y Naturales. Serie A. Matemáticas, vol. 114, p. 99, 2020.

[19] C. Izuchukwu, G. N. Ogwo, A. A. Mebawondu, and O. T. Mewomo, "On finite family of monotone variational inclusion problems in reflexive Banach space," University Politehnica of Bucharest Scientific Bulletin, vol. 82, pp. 89-104, 2020.

[20] S. Rezapour and S. H. Zakeri, "Hybrid method for equilibrium problems and variational inclusions," Journal of Inequalities and Applications, vol. 2020, p. 190, 2020.

[21] M. Wen, C. S. Hu, A. A. Cui, and J. G. Peng, "Algorithms for finding a common element of the set of common fixed points for nonexpansive semigroups, variational inclusions and generalized equilibrium problems," Revista de la Real Academia de Ciencias Exactas, Fisicas y Naturales-Serie A: Matematicas, vol. 114, p. 175, 2020.

[22] Y. T. Yang, "A viscosity method for treating variational inequality and variational inclusion problems," Journal of Nonlinear and Convex Analysis, vol. 21, pp. 923-931, 2020.

[23] Y. Yao, Y. C. Liou, and J. C. Yao, "Split common fixed point problem for two quasi-pseudo-contractive operators and its algorithm construction," Fixed Point Theory and Applications, vol. 2015, p. 127, 2015.

[24] Y. Yao, M. Postolache, and J. C. Yao, "An iterative algorithm for solving the generalized variational inequalities and fixed points problems," Mathematics, vol. 7, p. 61, 2019.

[25] C. Zhang, Q. L. Dong, and J. Chen, "Multi-step inertial proximal contraction algorithms for monotone variational inclusion problems," Carpathian Journal of Mathematics, vol. 36, pp. 159-177, 2020.

[26] C. Zhang, Z. Zhu, Y. Yao, and Q. Liu, "Homotopy method for solving mathematical programs with bounded box-constrained variational inequalities," Optimization, vol. 68, pp. 2293-2312, 2019.

[27] X. Zhao, J. C. Yao, and Y. Yao, "A proximal algorithm for solving split monotone variational inclusions," University Politehnica of Bucharest Scientific Bulletin-Series A-Applied Mathematics and Physics, vol. 82, no. 3, pp. 43-52, 2020.

[28] X. Zhao, D. R. Sahu, D. R. Sahu, and C.-F. Wen, "Iterative methods for system of variational inclusions involving accretive operators and applications," Fixed Point Theory, vol. 19, no. 2, pp. 801-822, 2018.

[29] L. C. Ceng and C. F. Wen, "Implicit hybrid steepest-descent methods for generalized mixed equilibria with variational inclusions and variational inequalities," Journal of Nonlinear and Convex Analysis, vol. 17, pp. 987-1012, 2016.

[30] D. Li and J. Zhao, "Approximation of solutions of quasivariational inclusions and fixed points of nonexpansive mappings," Journal of Nonlinear Sciences and Applications, vol. 9, no. 5, pp. 3002-3009, 2016.

[31] H. Zhou, "Strong convergence of an explicit iterative algorithm for continuous pseudo-contractions in Banach spaces," Nonlinear Analysis: Theory, Methods \& Applications, vol. 70, no. 11, pp. 4039-4046, 2009.

[32] S. Takahashi, W. Takahashi, and M. Toyoda, "Strong convergence theorems for maximal monotone operators with nonlinear mappings in Hilbert spaces," Journal of Optimization Theory and Applications, vol. 147, no. 1, pp. 27-41, 2010.

[33] H.-K. Xu, "Iterative algorithms for nonlinear operators," Journal of the London Mathematical Society, vol. 66, no. 1, pp. 240-256, 2002. 